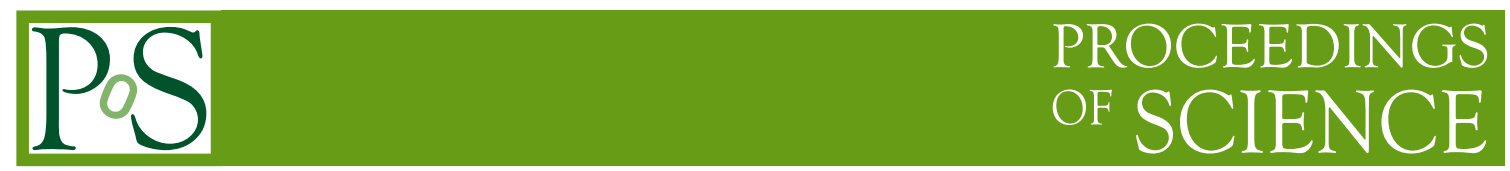

\title{
The SOX experiment hunts the sterile neutrino
}

Lea Di Noto ${ }^{* a}$, M. Agostini ${ }^{b}$, K. Altenmüller ${ }^{c}$, S. Appel ${ }^{c}$, V. Atroshchenko ${ }^{d}$, Z. Bagdasarian ${ }^{e}, f$, D. Basilico ${ }^{g}$, G. Bellini ${ }^{g}$, J. Benziger $^{h}$, D. Bick ${ }^{i}$, G. Bonfini $^{j}$, D. Bravo ${ }^{g}, k$, B. Caccianiga ${ }^{g}$, F. Calaprice ${ }^{l}$, A. Caminata ${ }^{a}$, S. Caprioli ${ }^{g}$, M. Carlini $^{j}$, P. Cavalcante ${ }^{j, k}$, A. Chepurnov ${ }^{m}$, K. Choi ${ }^{n}$, O. Clouée $^{o}$, L. Collica ${ }^{g}$, M. Cribier $^{o}$, D. D’Angelo ${ }^{\prime}$, S. Davini ${ }^{a}$, A. Derbin ${ }^{p}$, X.F. Ding ${ }^{b}$, A. Di Ludovicol ${ }^{l}$ I. Drachnev $^{b, p}$, M. Durero ${ }^{o}$, S. Farinon ${ }^{a}$, V. Fischer ${ }^{o}$, K. Fomenko ${ }^{q}$, A. Formozov ${ }^{g}, m, q$, D. Franco ${ }^{r}$, F. Gabriele j, J. Gaffiot ${ }^{o}$, C. Galbiati ${ }^{l}$, M. Gschwender ${ }^{s}$, C. Ghiano ${ }^{a}$, M. Giammarchi ${ }^{g}$, A. Goretti ${ }^{l}$, M. Gromov ${ }^{m}$, D. Guffanti ${ }^{b}$, C. Hagner ${ }^{i}$, Th. Houdy ${ }^{r}$, E. Hungerford ${ }^{t}$,

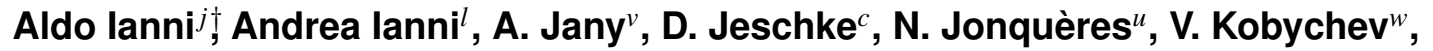
D. Korablev ${ }^{q}$, G. Korga ${ }^{t}$, V. Kornoukhov ${ }^{y}$, D. Kryn ${ }^{r}$, T. Lachenmaier ${ }^{s}$, T. Lasserre ${ }^{o}$, M. Laubenstein ${ }^{j}$, E. Litvinovich ${ }^{d, y}$, F. Lombardi ${ }^{j} ;$ P. Lombardi $^{g}$, L. Ludhova ${ }^{e, f}$, G. Lukyanchenko ${ }^{d}$, L. Lukyanchenko ${ }^{d}$, I. Machulin ${ }^{d, y}$, G. Manuzio ${ }^{a}$, S. Marcocci ${ }^{a, b}$, J. Maricic ${ }^{n}$, G. Mention ${ }^{o}$, J. Martyn ${ }^{z}$, E. Meroni ${ }^{g}$, M. Meyer ${ }^{a}{ }^{a}$, L. Miramonti ${ }^{g}$, M. Misiaszek ${ }^{v}$, V. Muratova ${ }^{p}$, R. Musenich ${ }^{a}$, B. Neumair ${ }^{c}$, L. Oberauer ${ }^{c}$, B. Opitz ${ }^{i}$, V. Orekhov ${ }^{d}$, F. Ortica ${ }^{a b}$, M. Pallavicini ${ }^{a}$, L. Papp ${ }^{c}$, Ö. Penek ${ }^{e, f}$, N. Pilipenko ${ }^{p}$,

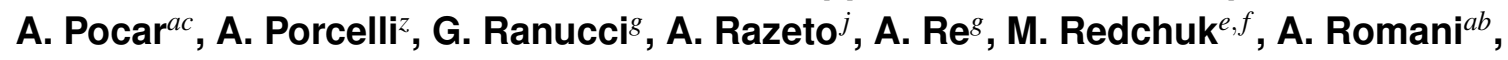
R. Roncin ${ }^{j, r}$, N. Rossi $^{j}$, S. Rottenanger ${ }^{s}$, S. Schönert ${ }^{c}$, L. Scola ${ }^{o}$, D. Semenov ${ }^{p}$, M. Skorokhvatov ${ }^{d, y}$, O. Smirnov ${ }^{q}$, A. Sotnikov $q$, L.F.F. Stokes ${ }^{j}$, Y. Suvorov ${ }^{d, a d}$, R. Tartaglia ${ }^{j}$, G. Testera ${ }^{a}$, J. Thurn ${ }^{a}$, M. Toropova ${ }^{d}$, E. Unzhakov ${ }^{p}$, C. Veyssiere ${ }^{o}$,

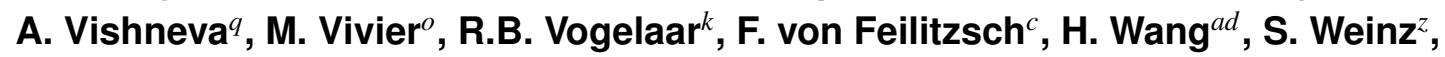
M. Wojcik ${ }^{v}$, M. Wurm ${ }^{z}$, Z. Yokley ${ }^{k}$, O. Zaimidoroga ${ }^{q}$, S. Zavatarelli ${ }^{a}$, K. Zuber $^{a}{ }$, G. Zuzel ${ }^{v}$ 
a Dipartimento di Fisica, Universitá degli Studi e INFN, Genova 16146, Italy

${ }^{b}$ Gran Sasso Science Institute (INFN), 67100 L'Aquila, Italy

${ }^{c}$ Physik-Department and Excellence Cluster Universe, Technische Universität München, 85748 Garching, Germany

${ }^{d}$ National Research Centre Kurchatov Institute, 123182 Moscow, Russia

e IKP-2 Forschungzentrum Jülich, 52428 Jülich, Germany

${ }^{f}$ RWTH Aachen University, 52062 Aachen, Germany

${ }^{g}$ Dipartimento di Fisica, Universitá degli Studi e INFN, 20133 Milano, Italy

${ }^{h}$ Chemical Engineering Department, Princeton University, Princeton, NJ 08544, USA

${ }^{i}$ Institut für Experimentalphysik, Universität, 22761 Hamburg, Germany

${ }^{j}$ INFN Laboratori Nazionali del Gran Sasso, 67010 Assergi (AQ), Italy

${ }^{k}$ Physics Department, Virginia Polytechnic Institute and State University, Blacksburg, VA 24061, USA

${ }^{l}$ Physics Department, Princeton University, Princeton, NJ 08544, USA

${ }^{m}$ Lomonosov Moscow State University Skobeltsyn Institute of Nuclear Physics, 119234 Moscow, Russia

${ }^{n}$ Department of Physics and Astronomy, University of Hawaii, Honolulu, HI 96822, USA

${ }^{o}$ Commisariat à l'Énergie Atomique et aux Énergies Alternatives, Centre de Saclay, IRFU, 91191 Gif-sur-Yvette, France

p St. Petersburg Nuclear Physics Institute NRC Kurchatov Institute, 188350 Gatchina, Russia

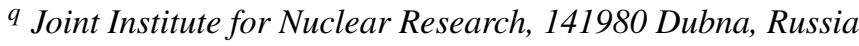

${ }^{r}$ AstroParticule et Cosmologie, Université Paris Diderot, CNRS/IN2P3, CEA/IRFU, Observatoire de Paris, Sorbonne Paris Cité, 75205 Paris Cedex 13, France

${ }^{s}$ Kepler Center for Astro and Particle Physics, Universität Tübingen, 72076 Tübingen, Germany

${ }^{t}$ Department of Physics, University of Houston, Houston, TX 77204, USA

u Commisariat à l'Énergie Atomique et aux Énergies Alternatives, Centre de Saclay, DEN/DM2S/SEMT/BCCR, 91191 Gif-sur-Yvette, France

${ }^{v}$ M. Smoluchowski Institute of Physics, Jagiellonian University, 30059 Krakow, Poland

${ }^{w}$ Kiev Institute for Nuclear Research, 03680 Kiev, Ukraine

${ }^{x}$ Institute for Theoretical and Experimental Physics, 117218 Moscow, Russia

${ }^{y}$ National Research Nuclear University MEPhI (Moscow Engineering Physics Institute), 115409 Moscow, Russia

${ }^{z}$ Institute of Physics and Excellence Cluster PRISMA, Johannes Gutenberg-Universität Mainz, 55099 Mainz, Germany

aa Department of Physics, Technische Universität Dresden, 01062 Dresden, Germany National Research Nuclear University MEPhI (Moscow Engineering Physics Institute), 115409 Moscow, Russia

${ }^{a b}$ Dipartimento di Chimica, Biologia e Biotecnologie, Universitá e INFN, 06123 Perugia, Italy

${ }^{a c}$ Amherst Center for Fundamental Interactions and Physics Department, University of Massachusetts, Amherst, MA 01003, USA

ad Physics and Astronomy Department, University of California Los Angeles (UCLA), Los Angeles, California 90095, USA

Email: lea.dinoto@ge.infn.it 
The SOX (Short distance neutrino Oscillations with BoreXino) experiment aims to perform a resolutive measurement for testing the longstanding hypotesis of a sterile neutrino in the $\mathrm{eV}^{2}$ mass scale. A very intense and well calibrated ${ }^{144} \mathrm{Ce}-{ }^{144} \mathrm{Pr}$ antineutrino source will be placed under the large size and very low background Borexino detector at Laboratori Nazionali del Gran Sasso in Italy. Borexino demonstrated a such energy and position resolution that the disappearance experiment can be performed and the short distance oscillations might be directly observed. In this paper an overview of the key elements of the experiment is given and the expected sensitivity to determine the sterile neutrino mass is shown.

XVII International Workshop on Neutrino Telescopes

13-17 March 2017

Venezia, Italy

\footnotetext{
* Speaker.

$\dagger$ Also at: Laboratorio Subterráneo de Canfranc, Paseo de los Ayerbe S/N, 22880 Canfranc Estacion Huesca, Spain

${ }^{\ddagger}$ Present address: Physics Department, University of California, San Diego, CA 92093, USA
} 


\section{Introduction}

Despite the standard three-flavour neutrino oscillation framework, in which the squared mass differences between the mass states are well under $0.01 \mathrm{eV}^{2}$, explains many experimental results mostly reported by solar [1, 2, 3] and atmospheric [4] neutrino experiments, a new type of neutrino was suggested by a small subset of data and its existence is not yet clarified. In fact some anomalous results have been reported in LSND [5] and MiniBooNE [6] experiments, where electron antineutrinos/neutrinos were found in a pure muon antineutrino/neutrino beam as well as in radioactive source experiments as GALLEX [7] and SAGE [8] that have evidenced a small deficit of neutrinos coming from ${ }^{51} \mathrm{Cr}$ and ${ }^{37} \mathrm{Ar}$ sources, respectively. In addition event rates measured by many reactor experiments at short distances demonstrated the disappearance of $\bar{v}_{e}$ [9], even if the evaluation of the reactor antineutrino fluxes is nowadays under discussion [10].

The combined analysis of all the anomalies [11,12], evidence that the data can be explained by introducing one or two additional sterile neutrinos that mixe with the three active neutrinos, calling for physics beyond the Standard Model. The global fit performed on the experimental results [13] suggests that the new mixing parameters are in the $\Delta m_{\text {new }}^{2} \sim 0.1-1 \mathrm{eV}^{2}$ and $\sin ^{2}\left(2 \theta_{\text {new }}\right) \sim 0.1$ region, implying that short distance oscillations with length less than $10 \mathrm{~m}$, if the neutrino energy is in the $1-10 \mathrm{MeV}$ region, has to be investigated in order to clarify the sterile neutrino existence. In general the short distance neutrino oscillation can be directly detected by means of neutrino/antineutrino beams coming from accelerators $[14,15]$ or reactors $[16]$ or by producing artificial sources. More recent experimental results from reactor experiments [16] or from indirect measurements [17] have reduced the allowed parameter space for a single sterile neutrino, but the direct measurement in the $\Delta m_{\text {new }}^{2}=0.1-1 \mathrm{eV}^{2}$ range have been not yet performed and this is the goal of the SOX experiment [18].

By exploiting the Borexino detector at Laboratori Nazionali del Gran Sasso and a very intense ${ }^{144} \mathrm{Ce}-{ }^{144} \mathrm{Pr}$ artificial antineutrino source a conclusive measurement may probe the reactor and gallium anomalies in a clear way, either confirming or discarding the sterile neutrino hypotesis and the short distance neutrino oscillations.

Borexino is a large scale detector, unique in the world in term of radiopurity and thanks to a small pit, built at the construction time and located at $8.35 \mathrm{~m}$ from the center of the detector sphere, where the source can be deployed, the allowed parameter region suggested by the anomalies can be investigate and the short distance oscillations in the sterile neutrino might be directly observed. The sensitivity of the measurement is based either on the detector performances, as the position and the energy resolution, or on the source properties knowledge, as the activity and the antineutrino energy spectrum and both have to be known with a very high precision.

In the following a detailed description of the detector and of the source will be reported and the activity measurement necessary for achieving the required sensitivity will be described. Finally the expected sensitivity plot in the $\Delta m^{2}-\sin ^{2}(2 \theta)$ parameters space will be shown.

\section{The source}

A ${ }^{144} \mathrm{Ce}-{ }^{144} \mathrm{Pr}$ source of about $120 \mathrm{kCi}$ activity is now in production at the Russian company "Federal State Unitary Enterprise Mayak Production Association". 
Through a long process of extraction, separation and purification from spent nuclear fuel, few kilograms of $\mathrm{CeO}_{2}$ powder containing a few tens of grams of ${ }^{144} \mathrm{Ce}$ (enough for the final source activity) are pressed up to a density of $3-5 \mathrm{~g} / \mathrm{cm}^{3}$ and inserted in a double wall stainless steel capsule. Then the capsule is placed in a cylindrical high density ( $95 \%$ tungsten, density $>18 \mathrm{~g} / \mathrm{cm}^{3}$ ) biological shield, whose thick (minimum $19 \mathrm{~mm}$ ) guarantees a $10^{12}$ attenuation factor for the gammas of $2.185 \mathrm{MeV}$ emitted by the ${ }^{144} \mathrm{Pr}$ decay with $0.7 \%$ branching ratio. The shield is finally closed with an helicoflex gasket and then transported by ship and truck from Russia to Italy in about one month.

The radioactive purity requirements of the source have been fixed in term of maximum gamma emitter activity that has to be less than $10^{-3} \mathrm{~Bq} / \mathrm{Bq}$ with respect to ${ }^{144} \mathrm{Ce}$ activity and in term of the ${ }^{244} \mathrm{Cm}$ activity (for neutron background) that has to be less than $10^{-5} \mathrm{~Bq} / \mathrm{Bq}$ with respect to ${ }^{144} \mathrm{Ce}$. In addition a limit is put on the activity of several nuclides that will be measured by the Russian company by alpha and gamma spectroscopy or by (ICP-MS) mass spectroscopy before the delivery and also on the ratio between the power released by the impurities, that has to be lower than $10^{-3}$ W/W respect with that released by the Cerium.

The contract with the Russian company was signed at the end of 2016 and the source will be delivered at St. Petersburg by 31th March 2018.

The ${ }^{144} \mathrm{Ce}-{ }^{144} \mathrm{Pr}$ pair is well suitable for SOX [19] since the $C e$ lifetime $\tau_{C e}=411$ days is long enough for the transportation and the data taking and the antineutrinos emitted by the ${ }^{144} \mathrm{Pr}$ beta-decay (with short lifetime $\tau_{P r}=24.94 \mathrm{~min}$ ), have an end-point energy of $3 \mathrm{MeV}$, higher the Borexino threshold of $1.8 \mathrm{MeV}$ (see section 3).

For the final sensitivity, not only the activity of the source has to be measured with high accuracy (better than 1\%), but also the ${ }^{144} \mathrm{Ce}$ and ${ }^{144} \mathrm{Pr}$ beta spectra have to be known, since both present non-unique forbidden transitions, for which the spectral shape theoretical predictions are uncertain at the few $\%$ level and past beta spectrum measurements show discrepancies at $10 \%$ level. Regarding the activity measurement, two different calorimeters have been built and calibrated (see section 5) in order to characterize the source just before the insertion in the tunnel, while for beta spectrum shape, new measurements for achieving about 3\% uncertainties are planned inside the collaboration, by developing dedicated apparatuses or by using the PERKEO spectrometer installed in Munich [20].

\section{The detector}

The ultra low background Borexino detector [21], located deep underground under a 3800 meter water equivalent was mainly designed for the observation and study of solar [22] and geo neutrinos [23]. A schematic layout is shown in Figure 1. The core of the detector is formed by 278 tons of ultra pure organic liquid scintillator contained in a transparent nylon spherical vessel $(\mathrm{R}=4.25 \mathrm{~m})$, surrounded by 2122 photomultipliers installed on a bigger stainless steel sphere of $6.85 \mathrm{~m}$ radius. A non-scintillating and transparent liquid fills the $1300 \mathrm{~m}^{3}$ space between the inner vessel and the stainless steel sphere, acting as shield against the external background. The stainless steel sphere is contained in a cylindrical tank filled with ultra-pure water, acting as a muon veto, thanks to 208 PMTs that detect the Cherenkov light of muon and as a passive shield against external gammas and neutrons, at a same time. During the installation special techniques were adopted for 


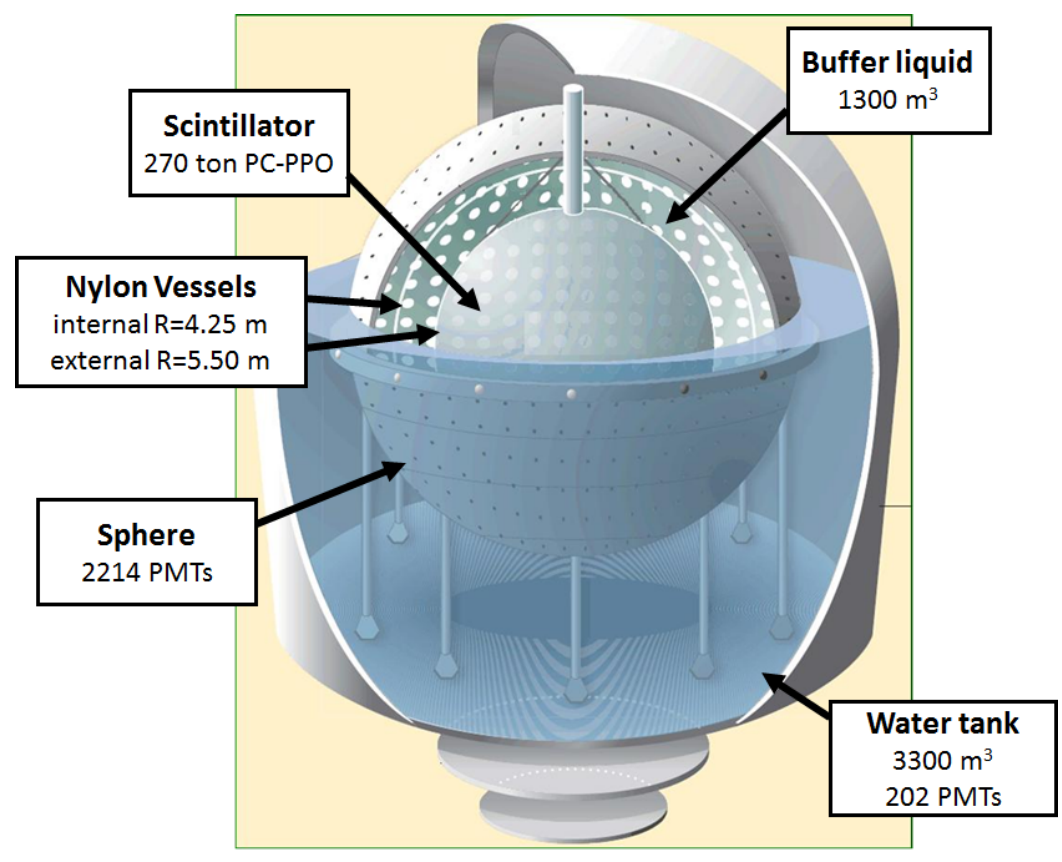

Figure 1: Borexino detector layout

the cleanness of each component [24], in such a way that Borexino reached an unprecedented level of radiopurity that results in an extremely low background allowing precise measurement of the solar neutrino fluxes [22] as well as of the geo-antineutrinos fluxes [25].

Borexino can detect antineutrinos thanks to the inverse beta-decay process (whose threshold is 1.8 $\mathrm{MeV}$ ) that produces coincidence events between the prompt positron annihilations and the $2.2 \mathrm{MeV}$ gamma emitted after the delayed neutron capture on protons. Due to the high activity of the source compared with the geoneutrino or reactor antineutrino fluxes (10 events/year), the data are almost background free and a statistic of $10^{4}$ events is expected in 1.5 year of data taking.

Borexino demonstrated a spatial resolution on the vertex reconstruction of about $12 \mathrm{~cm}$ and an energy resolution of about 3.5\% for antineutrino of $2 \mathrm{MeV}$ [3, 25]. However a new calibration campaign with neutrons, positron and gamma radioactive sources is scheduled before the SOX data taking, in order to confirm or possibly improve the spatial and energy resolution.

\section{Sterile neutrino signal}

The sterile neutrino evidence might emerge as deficit in the observed antineutrino rate ("disappearance technique") or as direct observation of the oscillation pattern ("waves"). In the first method the number of the observed antineutrino inside a fiducial volume has to be compared with the expected one and a deviation or deficit from $1 / r^{2}$ behavior can be interpreted as an oscillation in the sterile neutrino. In this case the sensitivity to the sterile neutrino mostly depends on the source activity accuracy, on the beta spectrum knowledge and on the fiducial volume estimation accuracy (see section 6). On the other hands there is the possibility that the waves might be directly observed in the L/E scale [26] and that the oscillation parameters $\Delta m_{\text {new }}^{2}$ and $\sin ^{2}\left(2 \theta_{\text {new }}\right)$ could be extracted independently, giving a clear confirmation of the sterile neutrino hypotesis. In this latter case the 
sensitivity to the wave detection is mainly related to the vertex reconstruction accuracy and to the detector energy resolution.

\section{The calorimetric measurements}

Since the disappearance technique is based on the knowledge of the ${ }^{144} \mathrm{Ce}$ activity, a precise measurement will be performed just before the source insertion under the Borexino tunnel by means of two high precision calorimeters appositely developed and calibrated by the CEA and the INFNTUM group within the SOX collaboration. Both the apparatus are conceived to extract the activity by measuring the power released in the shield and absorbed by a water flow and differently from the CEA calorimeter, where the water is in contact with the tungsten alloy shield, in the INFNTUM calorimeter the water flows inside a pipe brazed on a copper heat exchanger, in contact with the tungsten alloy shield. In both the systems, if the heat losses can be neglected, the power $P$ is achieved by measuring with high accuracy the mass flow $\dot{m}$ and the temperatures $T_{\text {out }}$ and $T_{\text {in }}$ of the water outgoing and entering in the system, according to the relation:

$$
P=\dot{m}\left[h\left(T_{\text {out }}, p\right)-h\left(T_{\text {in }}, p\right)\right]
$$

where $h(T, p)$ is the enthalpy of the water, which depends on the temperature and the average pressure inside the water line. Since the enthalpy function is tabulated by the International Association for the Properties of Water and Steam [27] and since the dependency on the pressure is really low, the pressure measurement can be performed at few percent level without any relevant influence on the final measured power. In both the apparatus the heat losses are minimized by using suspension system for reducing conduction, a shield of multilayer superinsulator materials to limit the radiative losses and a vacuum vessel working at a pressure less than $10^{-4}$ mbar to prevent any convection effect. Since the final accuracy can be affected not only by the statistical uncertainty given by the massflow and temperature measurements accuracy, but mainly by the systematic uncertainties due to the heat losses, many calibrations measurements have been planned before the source arrival, by using electrical heaters in a copper cylinder (as a mockup source), placed inside the tungsten alloy shield. The construction of both the calorimeters have been already completed and the calibrations are almost finished.

Regarding the INFN-TUM calorimeter, the calibration results, performed by applying to the heaters a constant and well known power, demonstrated that, under optimized conditions, the losses are always less than $0.2 \%$ and compatible with zero since the final statistical uncertainties of $0.2 \%$ resulted. In Figure 2 the measured power as a function of the time is shown, as acquired when a decaying power with the lifetime equal to the Cerium lifetime was set to the electrical heater. After an initial phase of around 2 days, necessary to the system to thermalize (that is present also when the power is set constant), the calorimetric measured value becomes close to the set value and after about 3 days its behavior follows the exponential function of the set power with the same time constant. In fact after the thermalization phase, the difference between the set and the measured power is found to be almost constant during the time and dependent not only on the heat losses (that would increase such difference), but mainly on the time necessary to the heat to be propagated from the inner source to the external copper jacket (that on the contrary decreases the difference itself). 


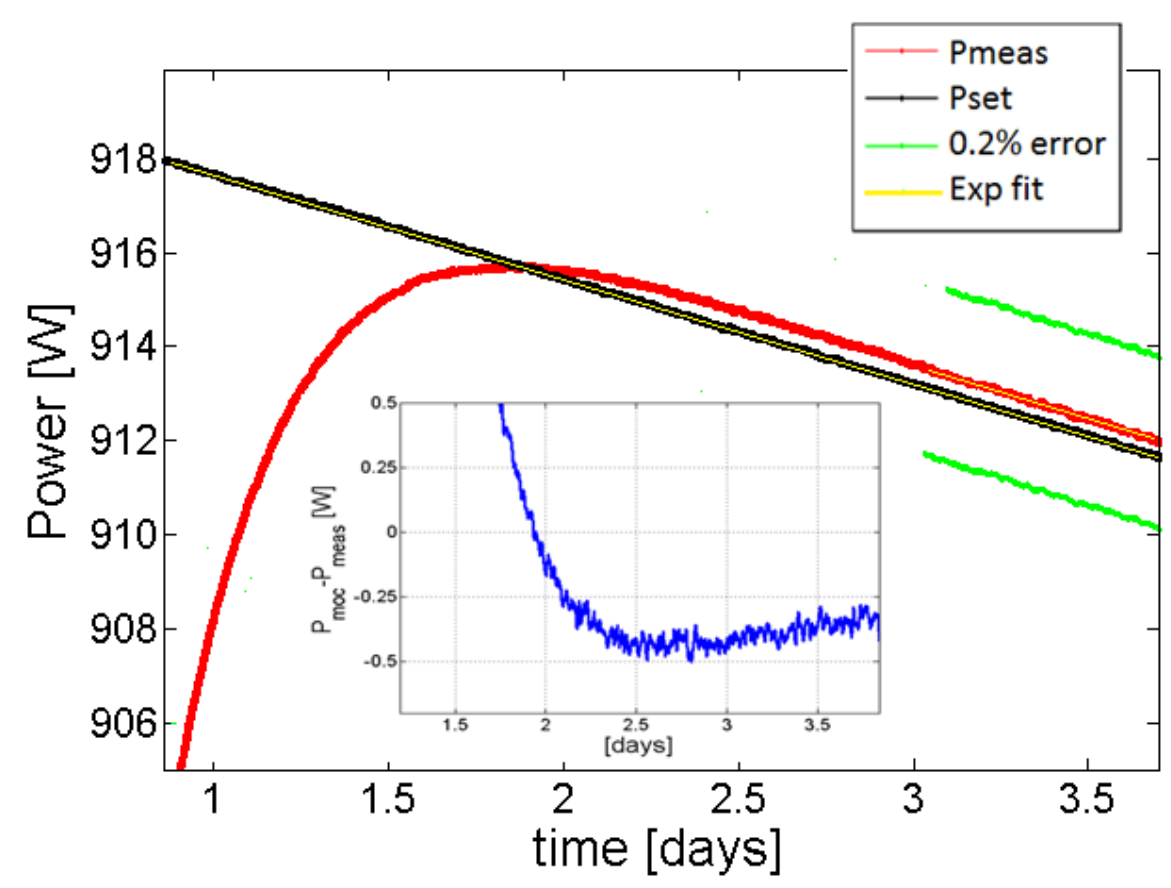

Figure 2: The measured power (in red) and the power applied to the electrical heater (in black) are shown as a function of the time. The yellow line corresponds to an exponential fuction with $\tau=\tau_{C e}$. The green line corresponds to the error band of $0.2 \%$ on the measured power.

However, since the Cerium lifetime is much bigger respect with this propagation time, the final difference between the set and the measured value after the 3 days of thermalization results inside the statistical error of $0.2 \%$ (see Figure 2), confirmating that the expected calorimetric measurement accuracy is about $0.2 \%$.

\section{SOX sensitivity}

The projected sensitivity of the SOX experiment at the 95\% C.L in the framework of a single additional sterile neutrino ( $3+1$ scenario), is shown on Figure 3. A 1.5 year of data taking was considered and the bandwidth is related to a the maximum $150 \mathrm{kCi}$ and minimum $100 \mathrm{kCi}$ value of the source activity. The assumed precision on the calorimetric measurement was $1.5 \%$, that is a very conservative number in comparison with the last calibration results obtained by the INFN-TUM calorimeter, while for the knowledge of the beta spectrum shape a value of $3 \%$ was considered. In the plot the contribution of the rate analysis and the shape analysis on the final sensitivity are shown and the evidenced region can be compared with the best fit region as resulted from the combined analysis of the antineutrino disappearance channel anomalies [13]. A wide area of the best fit region at 99\% C.L. is covered by the SOX sensitivity and thanks to the rate analysis the region is further enlarged.

\section{Conclusions}

The SOX project, here described, is a promising experiment for clarify the sterile neutrino 


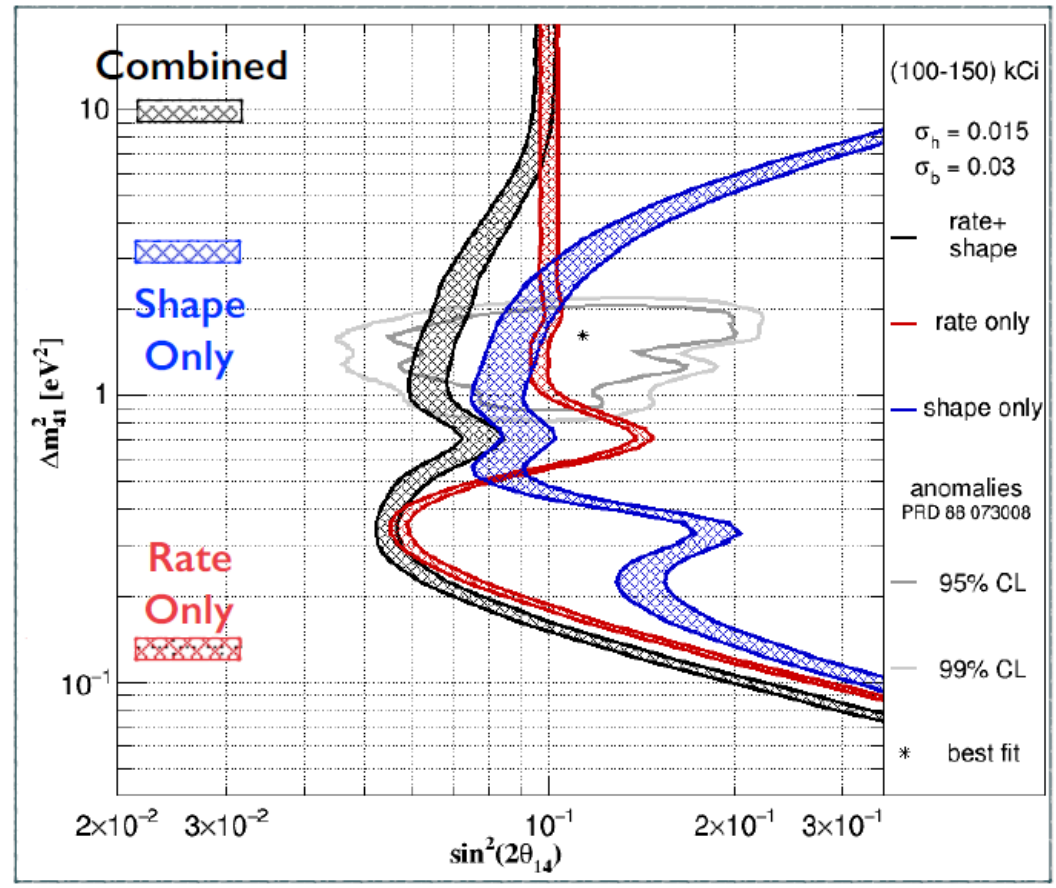

Figure 3: Sensitivity at the 95\% C.L. of the SOX experiment in the framework of a single additional sterile neutrino

puzzle in the $\Delta m^{2} \sim 0.1-1 e V^{2}$ range. A very intense artificial ${ }^{144} \mathrm{Ce}-{ }^{144} \mathrm{Pr}$ source is now in production and the calorimetric apparatus for the precise activity measurements have been calibrated and are ready for the final measurement, foreseen in May 2018. The SOX experiment is now close to the data taking and the first physics results are expected in late 2018 or 2019.

\section{References}

[1] S. Fukuda et al. (Super-KamiokaNDE-I Collaboration) Phys. Lett. B 539 (2002) 179-187

[2] Q. R. Ahmad et al. (SNO Collaboration), Phys. Rev. Lett. 89 (2002) 011301

[3] G. Bellini et al. (Borexino Collaboration) Phys. Rev. D 89 (2014) 112007

[4] Y. Fukuda et al. (Super-KamiokaNDE-I Collaboration) Phys. Rev. Lett. 81 (1998) 1562-1567

[5] A. Aguilar et al. (LSND Collaboration), Phys. Rev. D 64 (2001) 112007

[6] A. Aguilar et al. (MiniBooNe Collaboration), Phys. Rev. Lett. 110 (2013) 161801

[7] W. Hampel et al. (Gallex Collaboration), Phys. Lett. B 420 (1998) 114-126

[8] J. N. Abdurashitov et al. (SAGE Collaboration), Phys. Lett. B 328 (1994) 234-248

[9] G. Mention, M. Fechner, Th. Lasserre et al., Phys. Rev. D 83 (2011) 073006

[10] F.âĂL'P. An et al. (Daya Bay Collaboration) Phys. Rev. Lett. 118 (2017) 251801

[11] J. Kopp et al, JHEP 1305 (2013) 050

[12] C. Giunti and M. Laveder, Mod. Phys. Lett. A 22 (2007) 2499 
[13] C. Giunti et al, Phys. Rev. D 88, (2013) 073008

[14] C.M. Ignarra (MicroBooNE Collaboration), (2011) arXiv:1110.1604

[15] P. Adamson et al. (MINOS Collaboration), Phys. Rev. Lett. 117, (2016) 151803

[16] F.P. An et al. (Daya Bay Collaboration), Phys. Rev. Lett. 117, (2016) 151802

[17] M.G. Aartsen et al. (IceCube Collaboration), Phys. Rev. D 95, (2017) 112002

[18] G. Bellini et al (Borexino collaboration), JHEP 08 (2013) 038

[19] J. Gaffiot et al, Phys. Rev. D 91 (2015) 072005

[20] B. Märkisch et al, Nucl. Instr. Meth. A 611 (2009) 216-218

[21] G. Alimonti et al, Nuclear Instruments and Methods in Physics Research A 600 (2009) 568âĂŞ593

[22] G. Bellini et al. (Borexino Collaboration), Phys. Rev. D 89 (2014) 112007

[23] G.Bellini et al. (Borexino Collaboration), Phys. Lett. B, 687 (2010) 299.

[24] C. Arpesella et al. (Borexino Collaboration), Astropart. Phys. 18 (2002) 1

[25] M.Agostini et al, (Borexino collaboration), Physical Review D 92 (2015) 031101(R)

[26] Michel Cribier et al, Phys. Rev. Lett. 107, (2011) 201801

[27] International Association for the Properties of Water and Steam, Revised Release on the IAPWS Industrial Formulation 1997 for the Thermodynamic Properties of Water and Steam, R7-97 (2012), (http://www.iapws.org), 